\title{
Radiological Evaluation of Postoperative Complications after Non-Sleeve Gastrectomy Bariatric Procedures
}

\author{
Amgad S. Abdel-Rahman ${ }^{1}$, Ahmed Elshimy*1, Sayed H. M. Mohamed ${ }^{2}$ \\ ${ }^{1}$ Radiology Department, Faculty of Medicine, Ain Shams University, Cairo, Egypt \\ ${ }^{2}$ Radiology Department, National Liver Institute, Menoufia University, Menoufia, Egypt \\ *Corresponding author: Ahmed Elshimy, Mobile: (+20)01001294030, \\ E-mail: ahmedchimi@hotmail.com
}

\begin{abstract}
Background: Obesity is a medical condition, which may lead to serious related diseases, ultimately, resulting in many morbidities and early mortality. Its management involves many approaches of which bariatric surgery is considered nowadays as one of the most effective treatment for it. However, follow up of postoperative complications of this surgery by effective radiological method as computed tomography (CT) is important for assessment of its success.

Objective: The aim of this study was to illuminate the radiological signs and features of postoperative complications after non sleeve bariatric procedures and stressing the importance of using multi-slice CT (MSCT), and fluoroscopic study for detection of these complications. Patients and methods: An observational cohort study for 275 patients with suspected complications after non sleeve gastrectomy bariatric procedures, including 195 patients after Roux-en-Y gastric bypass (RYGB), 76 patients after laparoscopic adjustable gastric banding (LAGB) and 4 patients after intragastric balloon placement was done. These patients were subjected to either multi-slice CT and or fluoroscopy. Results: We detected complications in 21 patients out of the 195 patients who underwent RYGB: leakage, abscess, intestinal obstruction, internal hernia, port site ventral hernia, intussusception, fistula between the gastric pouch and the excluded stomach and hiatus hernia. On the other hand, 8 out of 76 patients operated by LAGB developed complications: band slippage, band erosion, pouch dilatation and tubal disconnection. Lastly two out of the 4 patients who placed intragastric balloon encountered other complications: gastric outlet obstruction, spontaneous balloon deflation and distal migration with intestinal obstruction.
\end{abstract}

Conclusion: It could be concluded that bariatric procedures may be followed by many complications and accurate diagnosis of these problems by proper radiological procedures as MSCT is imperative.

Keywords: Body mass index (BMI), Laparoscopic adjustable gastric banding (LAGB), Multi-slice CT (MSCT), Rouxen-Y gastric bypass (RYGB).

\section{INTRODUCTION}

Obesity is a multifactorial chronic illness, which showed an increasing prevalence all over the world in the last years ${ }^{(\mathbf{1}, 2)}$. In Egypt, it becomes a tremendous threat to health with $35 \%$ prevalence in adult population ${ }^{(3)}$, and up to $24 \%$ of children are also considerably overweight as published by Abdelkarim et al. in $2020^{(4)}$. The body mass index (BMI) is the most widely used measure of obesity; and it signifies overweight if between 25 and 30 , obesity if greater than 30 and morbid obesity if $>35$ with obesity related serious comorbidities or $>40$ with or without comorbidities ${ }^{(\mathbf{5}, \mathbf{6})}$.

Current treatment options for patients with obesity include lifestyle intervention, obesity pharmacotherapy, and bariatric surgery ${ }^{(7)}$.

Mainly three lines of surgical procedures are known for bariatric surgery: The first line with restrictive procedures including laparoscopic adjustable gastric banding (LAGB) and sleeve gastrectomy (SG), the second line with malabsorptive procedures including jejunoileal bypass and biliopancreatic diversion and the third line with combined restrictive and malabsorptive procedures including the Roux-en-Y gastric bypass (RYGB) ${ }^{(8)}$. RYGB and LAGB are the most commonly done procedures nowadays, followed by laparoscopic sleeve gastrectomy ${ }^{(9)}$.

In RYGB procedure, creation of a small gastric pouch and reducing the functional length of the jejunum is done. Complications from RYGB surgery include enteric leak/abscess, hemorrhage, gastrogastric fistula, anastomotic stricture, and small bowel obstruction ${ }^{(\mathbf{1 0})}$.

However, in LAGB, a silicon band is placed around the proximal stomach, approximately $2 \mathrm{~cm}$ below the gastroesophageal junction, creating a proximal pouch with a volume of $\sim 15-30 \mathrm{~mL}$ with diameter less than 4 $\mathrm{cm}$, which communicates to the remainder of the stomach through a narrowed adjustable stoma via a subcutaneous access port with a stomal diameter goal of 3-5 mm. Many complications can follow LAGB as slippage of band/gastric prolapse, gastric perforation from band erosion, stomal stenosis/pouch dilatation, and mechanical port/tubing complications as tubing disconnection and port rotation/inversion ${ }^{(11,12)}$.

Endoscopical intragastric balloon temporary placement is achieving more acceptance owing to its safety and effectiveness beside the simplicity of its procedure with lower cost and lacking the lifelong side effects. It restricts food intake by promoting early satiety and followed up until endoscopically removed 6 month later ${ }^{(13)}$. Its complications include enteric perforation and balloon migration with obstruction of the bowel which may necessitate emergent management and intervention $^{(14,15)}$.

Evaluation of suspected postoperative complications is important and could depend on radiographs, upper gastrointestinal (UGI) series, and CT 
scan. Fluoroscopic UGI series is considered the first imaging step and is routinely performed within the first 2 postoperative days, while $\mathrm{CT}$ is usually performed if extraluminal complications are suspected, especially with equivocal fluoroscopic studies ${ }^{(12)}$.

The aim of this study was to illuminate the radiological signs and features of postoperative complications after non sleeve bariatric procedures and stressing the importance of using multi-slice CT (MSCT), and fluoroscopic study for detection of these complications.

\section{PATIENTS AND METHODS}

This is an observational cohort study, including patients with suspected post non sleeve gastrectomy bariatric procedures complications, these patients were subjected to either multi-slice CT or fluoroscopy or both in Ain Shams University Hospitals_during period of 16 months (from June 2020 till September 2021) to assess the integrity of the procedures and detection of their complications. A total of 275 patients with suspected complications, 195 after RYGB, 76 after LAGB and 4 after intra-gastric balloon placement.

Inclusion criteria: Patients with suspected complications after non sleeve gastrectomy bariatric procedures.

Exclusion criteria: Compromised renal function (serum creatinine more than $1.5 \mathrm{mg} / \mathrm{dl}$ ) and allergy to iodinecontaining contrast material.

These patients underwent either upper GIT fluoroscopic study with oral contrast material or Multi-slice CT examination or both.

\section{Ethical approval:}

Written informed consent of all subjects was obtained after thoroughly explanation for them the protocol of the study. Approval of the Ethical Committee of the Radiology Department Scientific Board after following the ethical guidelines of Faculty of Medicine, Ain Shams University; Cairo - Egypt was obtained. This work has been carried out in accordance with The Code of Ethics of the World Medical Association (Declaration of Helsinki) for studies involving humans.

The technique of fluoroscopic upper gastrointestinal series (UGIS):

Performed using conventional X-ray fluoroscopy (GE Precision RXi). We used water soluble contrast medium in early postoperative period within the $1^{\text {st }} 30$ days and barium sulphate in late postoperative cases later. Supine control plain "scout" film was done first to assess the position of the surgical staples or the gastric band confirming its location and shape and assessing the phi (Ø) angle (normally should be between 4 and $58^{\circ}$ ), stomal caliber, port position, course and continuity of the connector tube. Then, we traced a swallowed bolus of contrast in supine position passing through lower esophagus into the stomach/gastric pouch till reaching the small intestine (duodenum/ jejunum), which would be through the gastrojejunal anastomosis into the Roux limb in cases of Roux-en-Y gastric bypass. Spot views were taken and we stopped the examination if leakage was noticed. To check for port and tube leak or disconnection, or band asymmetry the port was accessed with a needle and after draining its content, slowly injecting 5-10 $\mathrm{ml}$ of water-soluble contrast material to check of any leakage. Multiple spot views were taken. At the end of study, we withdrew the contrast and reinjected a sterile saline into the system equal to the original drained volume.

\section{The technique of multi-slice CT examination:}

The study was performed using two CT scanners, Toshiba PRIME Aquilion 164 slice, and Philips Brilliance 16 slice. A dedicated CT scan for each patient was done depending on his clinical data and plain CT findings. The whole CT protocol encompass noncontrast plain acquisition as a start, then followed by contrast enhanced acquisition after oral ingestion and IV injection of contrast. Patients were informed to ingest oral contrast as much as they can afford. IV injection of $100 \mathrm{ml}$ of non-ionic contrast (Omnipaque) was done through anti-cubital fossa 18 gauge cannula followed by injection of $30 \mathrm{ml}$ of saline using automatic injector at a rate of 3-4 ml/s, venous phase acquisition was taken with $75 \mathrm{sec}$ delay. Arterial and delayed phase scans were taken if needed. To assess for leak only in some patients, non-contrast plain scan was taken either with or without oral administration of contrast. Reformatted images and $3 \mathrm{D}$ reconstruction if needed were performed using Vitrea workstation. CT scans were evaluated separately by two radiologists with 5 years' experience in bariatric imaging and each one defined a diagnostic impression.

\section{Statistical analysis}

Basic demographic and postoperative radiological data were collected and entered into a database (Excel ${ }^{\circledR}$, Office Microsoft). Quantitative data were statistically described in terms of range, average, while qualitative data were presented as frequencies (number of cases) and percentages $(\%)$.

\section{RESULTS}

In this study, 275 patients were included and their ages ranged from 19 to 65 years with 42.4 years average age. Of these patients, 226 were females $(82.2 \%)$ and the other $49(17.8 \%)$ were males.

Out of the 275 included patients, 195 patients (70.9\%) had done RYGB and 21 of them (10.8\%) showed various types of postoperative complications, while the remaining 174 patients were free. Most of the complicated cases after RYGB procedure developed late complications (19 patients; 90.5\%).

On the other hand, 76 patients $(27.6 \%)$ had done LAGB and 8 of them (10.5\%) showed various types of complications and the remaining 68 patients were free. The remaining 4 patients had placed intragastric balloon came to ER with colicky pain and distention and 2 patients showed radiological findings for complication.

Among the 21 patients who developed complications after RYGB procedure, 16 were females $(76.2 \%)$ and 5 were males $(23.8 \%)$ and these complications are shown in table 1 and figures 1-4. 
Table (1): Post RYGB complication in our study with their percentages

\begin{tabular}{|l|c|c|}
\hline \multicolumn{1}{|c|}{ Complications } & No of cases & $\%$ \\
\hline Extraluminal leakage & $\mathbf{2 / 1 9 5}$ & $\mathbf{1 \%}$ \\
- From gastrojejunal anastomosis & $2 / 2$ & $100 \%$ \\
$>$ Developed abscess & $2 / 2$ & $100 \%$ \\
$>$ Developed fistula & $1 / 2$ & $50 \%$ \\
\hline Abscess (developed after Extraluminal leakage) & $\mathbf{2 / 1 9 5}$ & $\mathbf{1 \%}$ \\
\hline Intestinal obstruction & $\mathbf{4 / 1 9 5}$ & $\mathbf{2 \%}$ \\
$>$ Caused by internal hernia & $1 / 4$ & $\mathbf{2 5 \%}$ \\
$>$ Caused by port site hernia & $1 / 4$ & $\mathbf{2 5 \%}$ \\
$>$ Caused by intussusception & $2 / 4$ & $\mathbf{5 0 \%}$ \\
\hline Internal hernia & $\mathbf{1 4 / 1 9 5}$ & $\mathbf{7 . 2 \%}$ \\
- Led to intestinal obstruction & $1 / 14$ & $7.1 \%$ \\
$>$ Associated with intussusception & $1 / 14$ & $7.1 \%$ \\
\hline Port site hernia & $\mathbf{1 / 1 9 5}$ & $\mathbf{0 . 5 \%}$ \\
$>$ Incarcerated and led to intestinal obstruction & & \\
\hline Intussusception & $\mathbf{3 / 1 9 5}$ & $\mathbf{1 . 5 \%}$ \\
- Led to intestinal obstruction & $2 / 3$ & $66.7 \%$ \\
P Associated with internal hernia & $1 / 3$ & $33.3 \%$ \\
\hline Gastrojejunal anastomotic inflammation with marginal ulceration & $\mathbf{1 / 1 9 5}$ & $\mathbf{0 . 5 \%}$ \\
& & \\
\hline Fistula between the gastric pouch and the excluded stomach & $\mathbf{3 / 1 9 5}$ & $\mathbf{1 . 5 \%}$ \\
\hline Hiatus hernia & $\mathbf{3 / 1 9 5}$ & $\mathbf{1 . 5 \%}$ \\
\hline
\end{tabular}
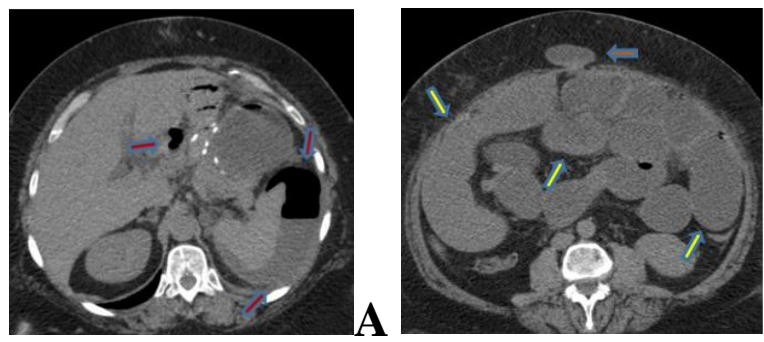

B
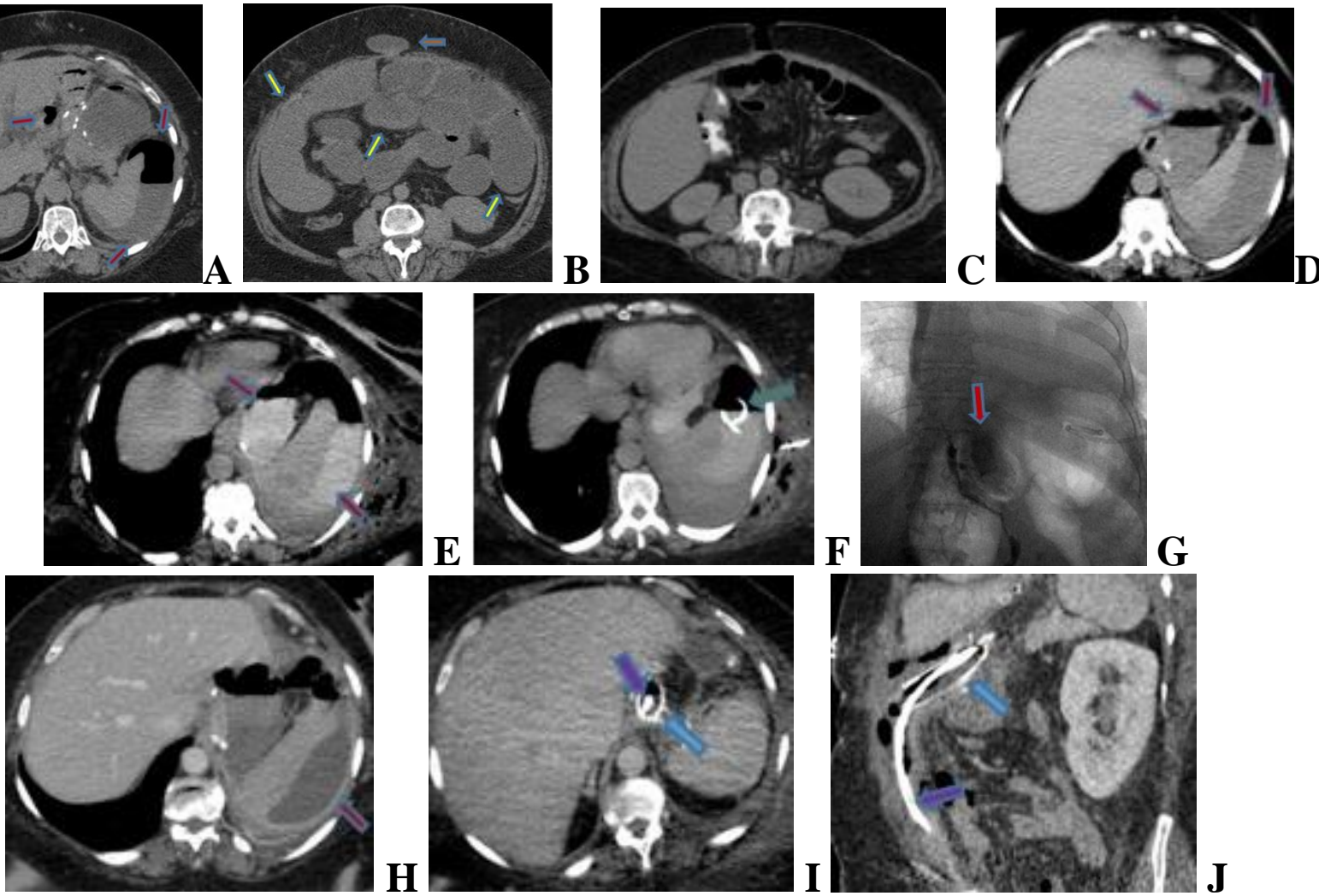

Figure (1): A 56 year old female patient underwent gastric bypass one day back, who experienced second day postoperative severe upper abdominal pain, vomiting and distention. (A and B) Postoperative axial CT images without contrast showing free fluid leakage (red arrows) with air fluid levels on the left aspect of the gastrojejunal anastomosis and left subphrenic region around the spleen with incarcerated port site hernia containing small bowel (orange arrow) and dilated fluid filled proximal small bowel loops (yellow arrows) and collapsed distal bowel loops. (C and D) Follow up axial CT images after release of the port site hernia showing decompressed small bowel loops with still noted free fluid leakage with air fluid levels (red arrows). (E and F) Follow up axial CT images with oral contrast after insertion of pig tail external drainage (green arrow) into left subphrenic space with notable related subcutaneous emphysema and still noted contrast leakage and fluid levels. (G) Follow up fluoroscopic image with oral water soluble contrast showing 
oral contrast leakage (red arrow). (H) Later follow up axial CT image with IV contrast injection showing persistent leakage with marginal enhancement (infected leak). (I and J) Follow up axial and sagittal oblique CT images after application of gastric stent (blue arrows) and nasojejunal tube (purple arrows) for feeding.
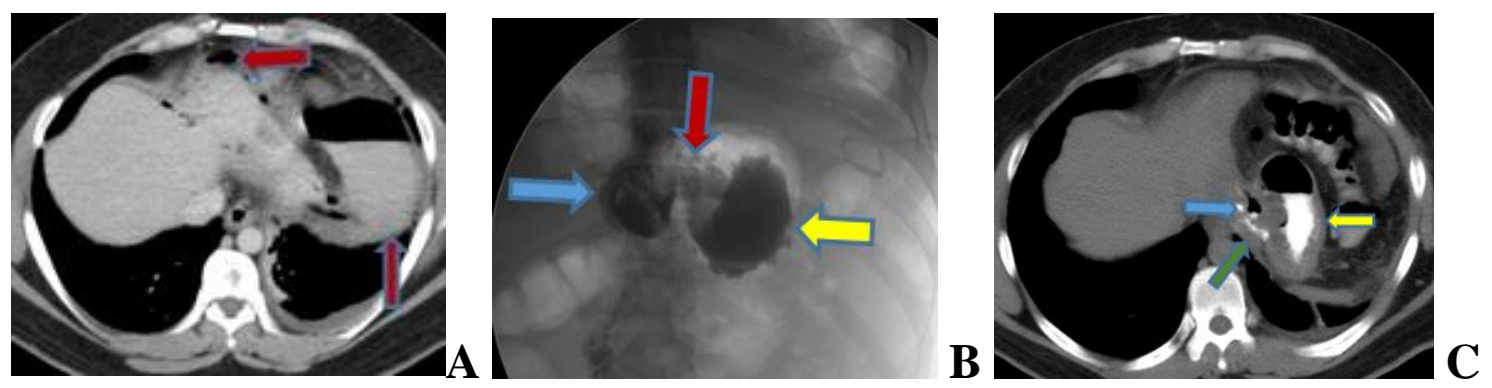

Figure (2): A 32 year old male patient underwent gastric bypass two days back who experienced severe upper abdominal pain. (A) Postoperative axial CT image after oral contrast ingestion showing left subphrenic extraluminal contrast locule and free air (red arrows). (B) Follow up fluoroscopic image with oral water soluble contrast done 20 days later showing extraluminal contrast locule (red arrow) seen between the opacified gastric pouch (blue arrow) and the opacified excluded stomach (yellow arrow) denoting fistulous communication, left hypochondrial pig-tail catheter is also noted. (C) Follow up axial CT image after oral contrast administration showing a fistulous tract (green arrow) between the opacified excluded stomach (yellow arrow) and the collapsed gastric pouch (blue arrow).
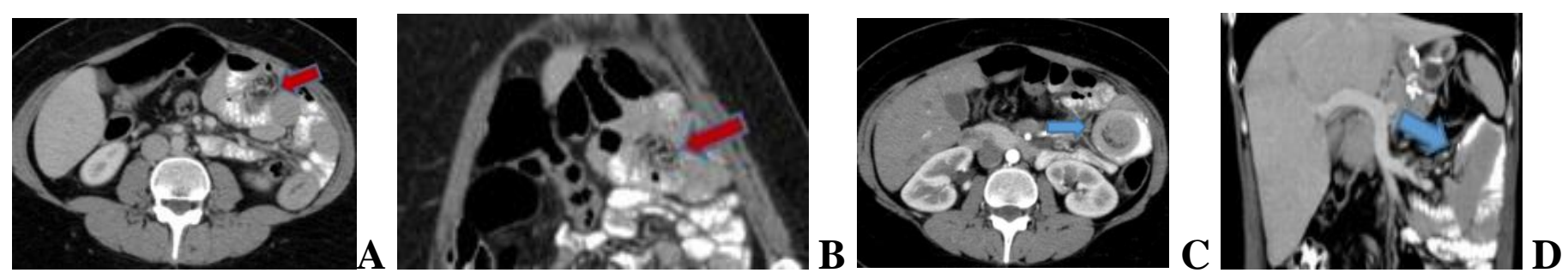

Figure (3): A 30 year old female patient underwent gastric bypass several years back who experienced colicky abdominal pain. (A and B) Post contrast CT axial and reformatted coronal oblique images showing internal hernia at left abdomen (red arrow) with characteristic vascular and small bowel loops swirl sign within a constricting neck. (C and D) Axial and coronal CT images showing intussusception (blue arrow) at the jejunojejunal anastomosis showing the characteristic target sign in the axial image.
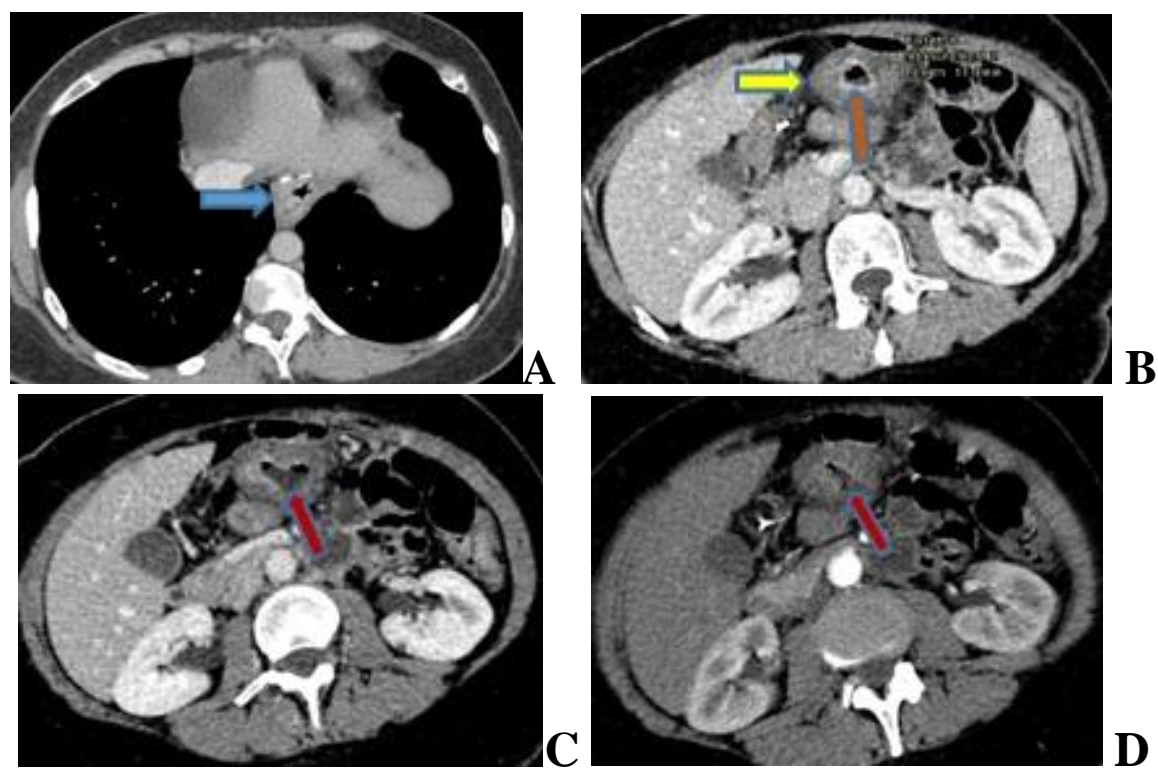

Figure (4): A 47 year old female patient underwent gastric bypass several years back, who experienced severe upper abdominal pain. (A) Post contrast CT axial image showing small hiatus hernia with stables seen above the diaphragm (blue arrow). (B, C and D) Post contrast CT axial images showing inflamed jejunal loop at the gastrojejunal anastomosis with circumferential wall thickening (yellow arrow) and enhancing mucosal line (orange arrow) that shows focal posterior mucosal defect seen in images $\mathrm{C}$ and $\mathrm{D}$ denoting ulceration (red arrow). 
Among the 8 patients who developed complications after laparoscopic adjustable gastric band (LAGB) placement, 7 were females (87.5\%) and 1 was male (12.5\%) and these various complications are shown in table 2 and figures 5-9.

Table (2): Post LAGB placement complication in our study with percentages

\begin{tabular}{|l|c|c|}
\hline \multicolumn{1}{|c|}{ Complications } & No of cases & \% \\
\hline Band slippage & $\mathbf{5 / 7 6}$ & $\mathbf{6 . 6 \%}$ \\
- Associated with gastric wall band erosion & $1 / 5$ & $20 \%$ \\
- Retro-peritoneal hematoma occurred after its removal. & $1 / 5$ & $20 \%$ \\
\hline Gastric wall band erosion & $\mathbf{1 / 7 6}$ & $\mathbf{1 . 3 \%}$ \\
- Associated with band slippage & & \\
\hline Pouch dilatation & $\mathbf{6 / 7 6}$ & $\mathbf{7 . 9 \%}$ \\
- Caused by band slippage & $5 / 6$ & $83.3 \%$ \\
- Without band slippage (overfilling) & $1 / 6$ & $16.7 \%$ \\
D Associated with esophageal dilatation & $1 / 6$ & $16.7 \%$ \\
\hline Tubal disconnection & $\mathbf{2 / 7 6}$ & $\mathbf{2 . 6 \%}$ \\
\hline
\end{tabular}
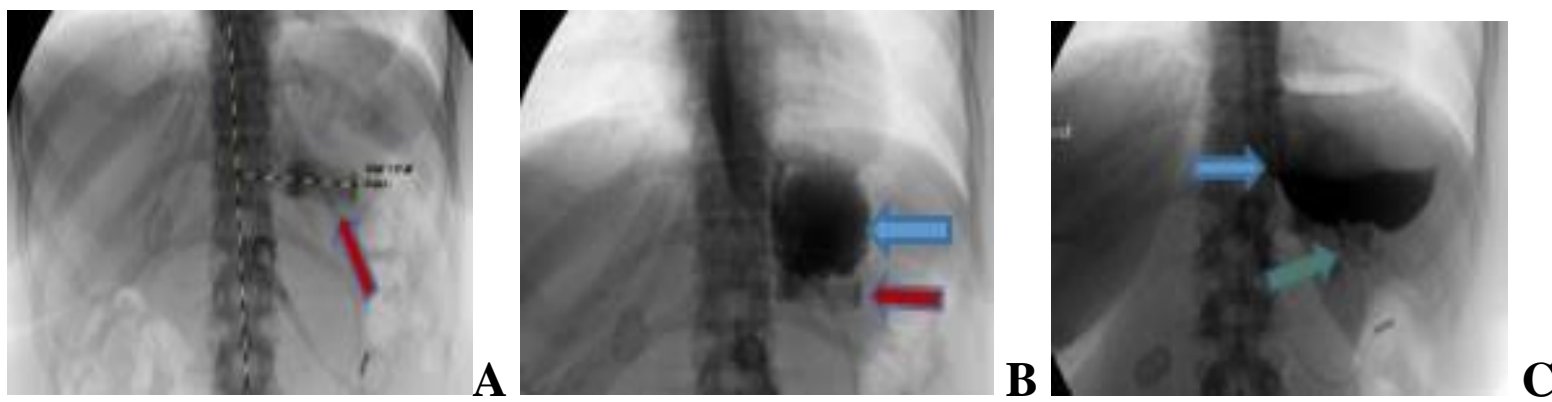

Figure (5): A 41 year old female patient with applied LAGB. (A, B and C) Fluoroscopic study images with oral water soluble contrast showing almost horizontal position of the band (red arrow) with phi angle $=97^{\circ}\left(\mathrm{N}\right.$. from $\left.4-58^{\circ}\right)$ with asymmetric pouch dilatation (blue arrow) and faint thread of contrast (green arrow) passed through the stoma only in upright position.
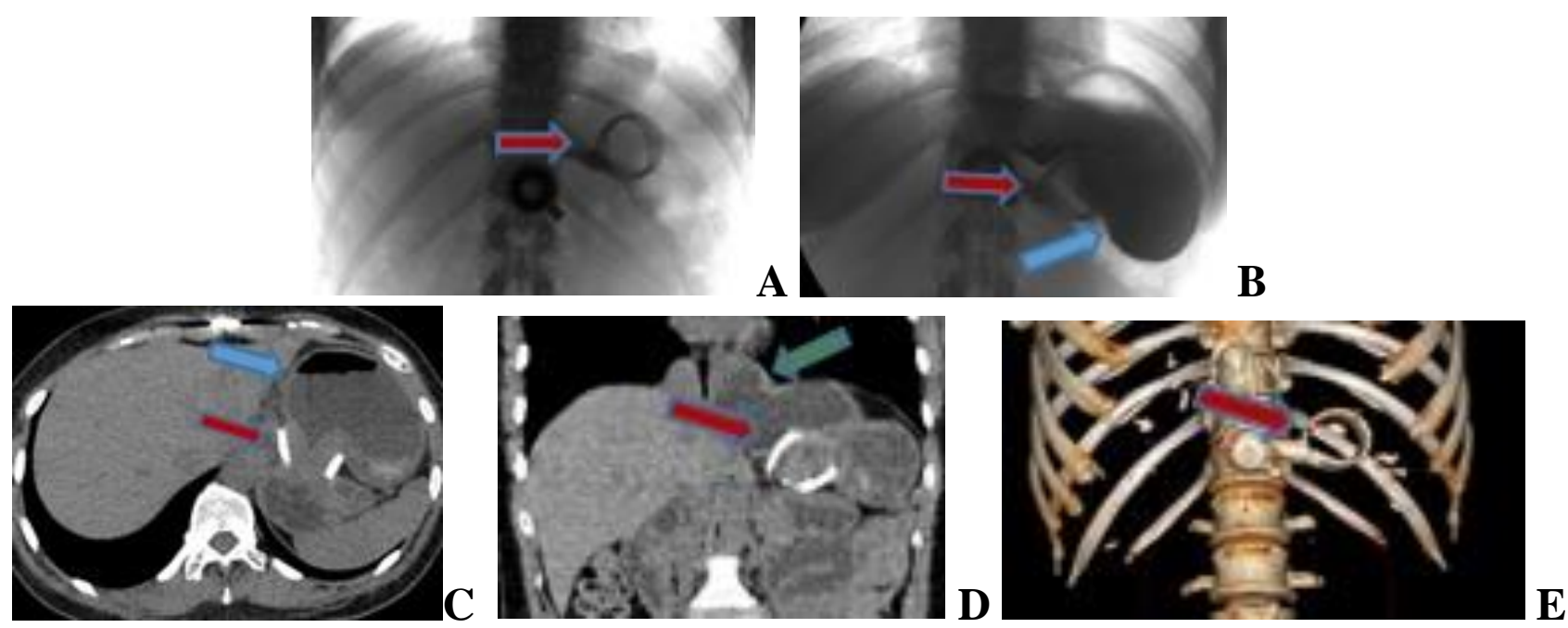

Figure (6): A 45 year old female patient with applied LAGB. (A and B) Fluoroscopic study images with oral water soluble contrast showing slipped band with abnormal orientation (red arrow) giving $\mathrm{O}$ sign being seen en face with asymmetric pouch dilatation (blue arrow) and no contrast passed through the stoma. (C and D) CT axial and coronal images showing the slipped and abnormally orientated band (red arrow) and asymmetric pouch dilatation (blue arrow) and a small hiatus hernia (green arrow). (E) 3D CT image showing the slipped and abnormally orientated band (red arrow) giving $\mathrm{O}$ sign being seen en face. 


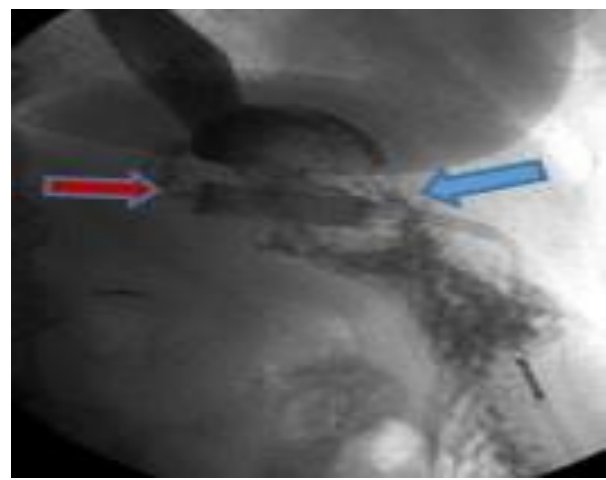

Figure (7): A 65 year old female patient underwent LAGB. Fluoroscopic study image with oral water-soluble contrast showing dislocated slipped gastric band (red arrow) with contrast noted outside the band (blue arrow) indicating gastric band erosion.
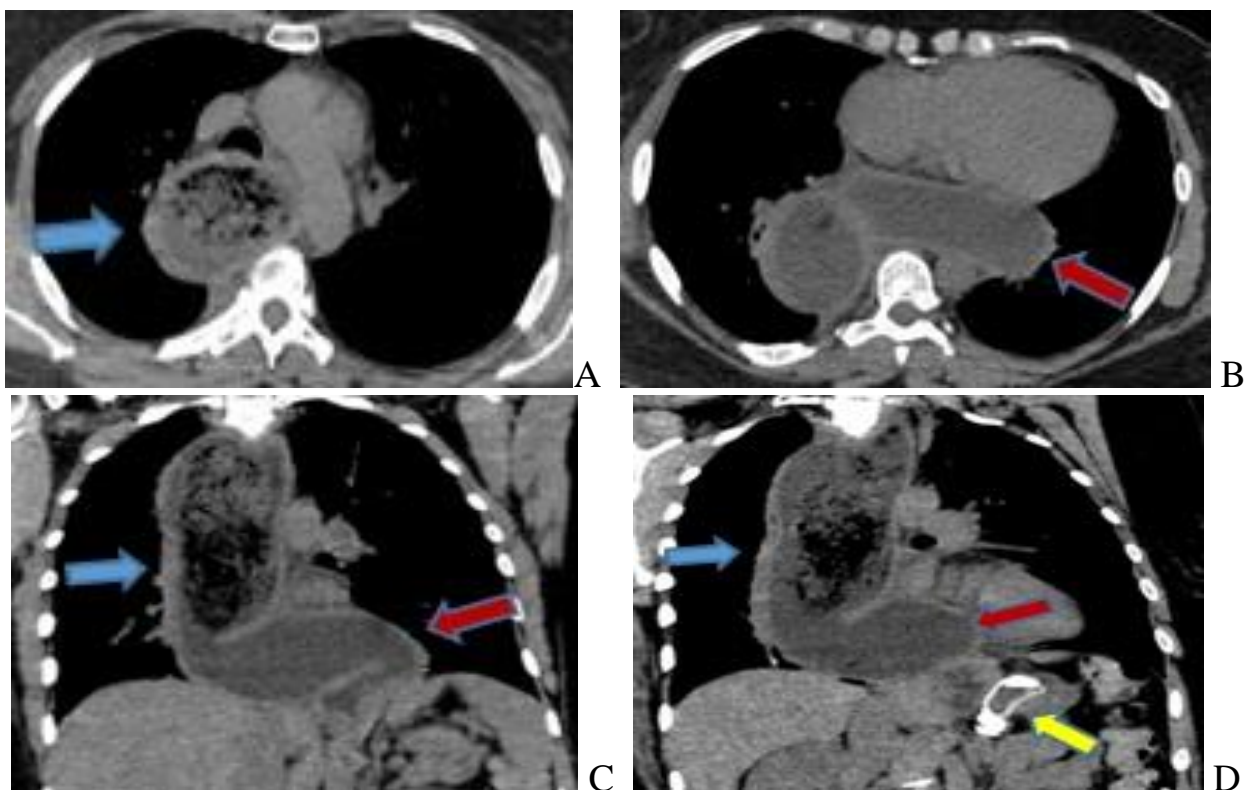

Figure (8): A 49 year old female patient underwent LAGB who experienced dysphagia and epigastric pain. (A and B) $\mathrm{CT}$ axial images and (C and D) Reformatted coronal and coronal oblique images showing the gastric band (yellow arrow) in its normal position and orientation, marked dilatation of the gastric pouch (red arrow) forming a large paraesophageal hiatus hernia and marked esophageal dilatation (blue arrow) reaching up to $7.5 \mathrm{~cm}$ in diameter with retained food particles (mega Esophagus; pseudo-achalasia).
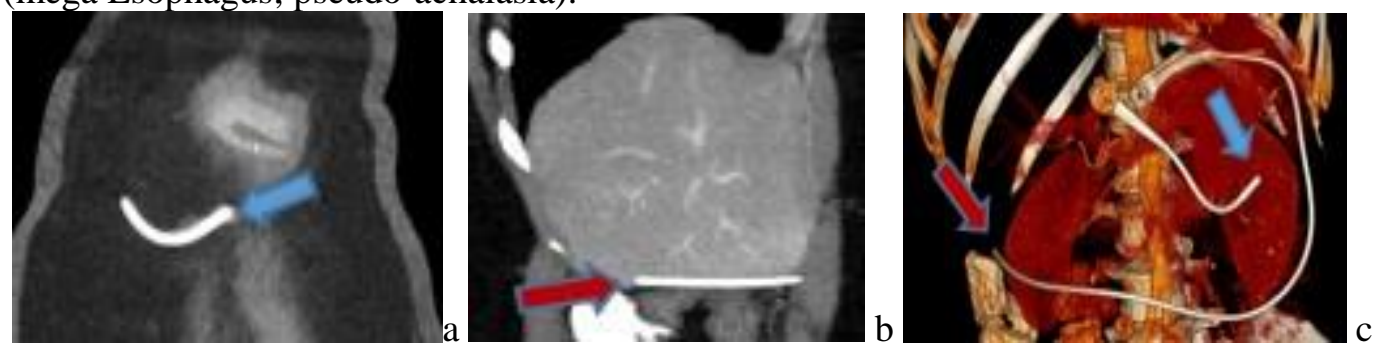

Figure (9): A 46 year old female patient underwent LAGB. (A and B) Reformatted coronal images showing line disconnection with proximal segment attached to the access port and runs in the subcutaneous tissue of left upper abdomen and ends blindly (blue arrow in a) and the distal segment attached to the gastric band ends blindly in the subhepatic region (red arrow in b). (C) 3D layout image best showing line disconnection.

Among the 4 patients who came to our hospital ER complaining from colicky pain with history of placed intragastric balloon, we observed the followings:

1. One patient was completely free on the radiological basis and the intra-gastric balloon was seen within the stomach, showing air fluid level and having $9.8 \mathrm{~cm}$ diameter with no signs of gastric outlet obstruction.

2. In the second case, the balloon was spontaneously deflated and distally migrated with a piece seen within the proximal ileum causing small bowel obstruction. The patient gave a history of intra-gastric balloon placement 10 months earlier and she had 2 days symptoms of severe colicky pain, vomiting and constipation with bluish discoloration of the urine (fig. 10).

3. In the third case, the intra-gastric balloon wasn't seen at all; neither within the stomach nor within bowel loops. The patient gave a history of intra-gastric balloon placement 14 months earlier and she had one day symptoms 
of severe colicky pain, vomiting and diarrhea with bluish discoloration of the urine. Based on all of these data and negative radiological finding, a suggestion of balloon spontaneous deflation and distal migration passing out of the alimentary canal was made with no residual complications.

4. In the fourth case; the intra-gastric fluid filled balloon was seen distending gastric antrum and had $9 \mathrm{~cm}$ diameter, the proximal stomach (gastric fundus and body) was moderately distended by the ingested water $(+950 \mathrm{cc})$ and showed air fluid level with no food particles (picture of gastric outlet obstruction secondary to the intragastric balloon). The patient gave history of intra-gastric balloon placement 9 months earlier and she came complaining of central and right upper abdominal pain, distension and discomfort with food intolerance and nausea for 10 days and recently experienced non bilious vomiting after eating (fig. 11).
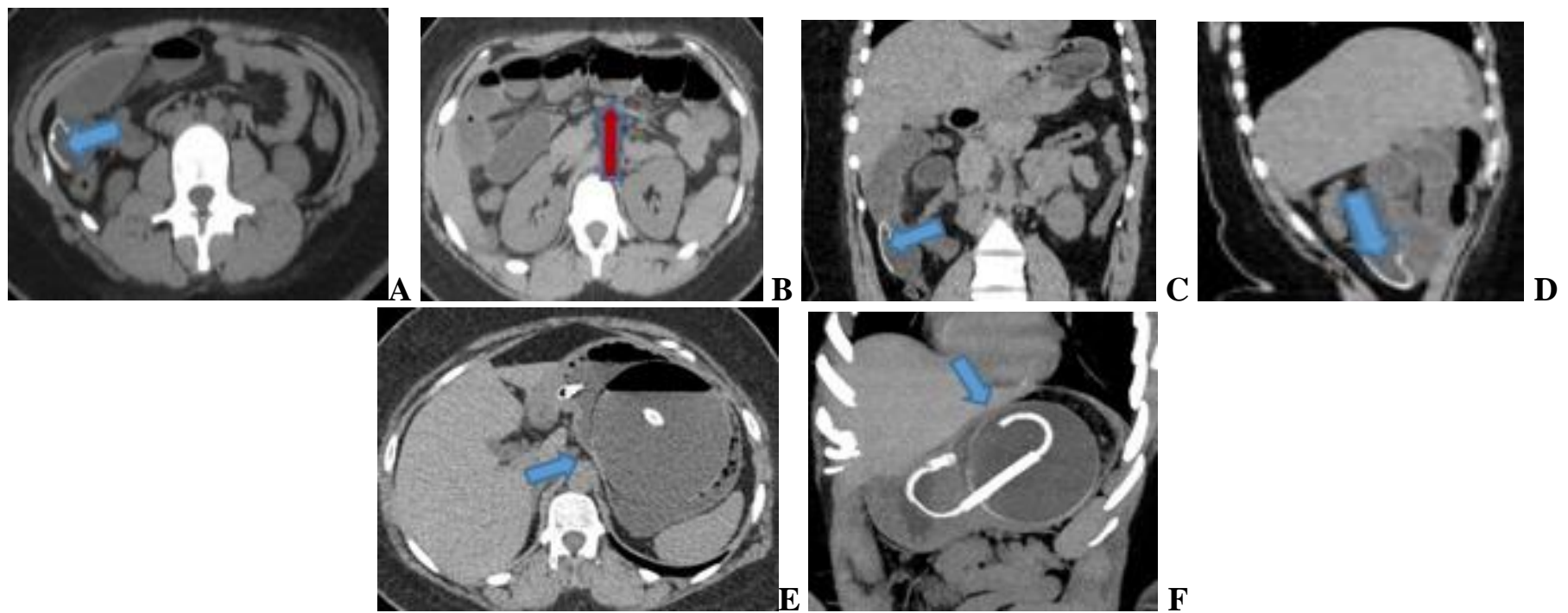

Figure (10): A 19 year old female patient with gastric balloon who experienced severe colicky abdominal pain, vomiting and distention with bluish discoloration of her urine for two days. (A and B) CT axial images showing distal migration of a piece of the spontaneously deflated gastric balloon which is seen within proximal ileal loop at the right lumbar region (blue arrows) with mild dilatation of the proximal bowel loops (red arrows) measuring about $33 \mathrm{~mm}$ in diameter filled with fluid and gas and the distal small bowel loops were collapsed. (C and D) reformatted coronal and sagittal oblique images showing the distally migrated piece of the spontaneously deflated gastric balloon. (E and F) Axial and reformatted coronal oblique MIP images showing normal CT appearance of a normal gastric balloon (blue arrows) in another normal patient.
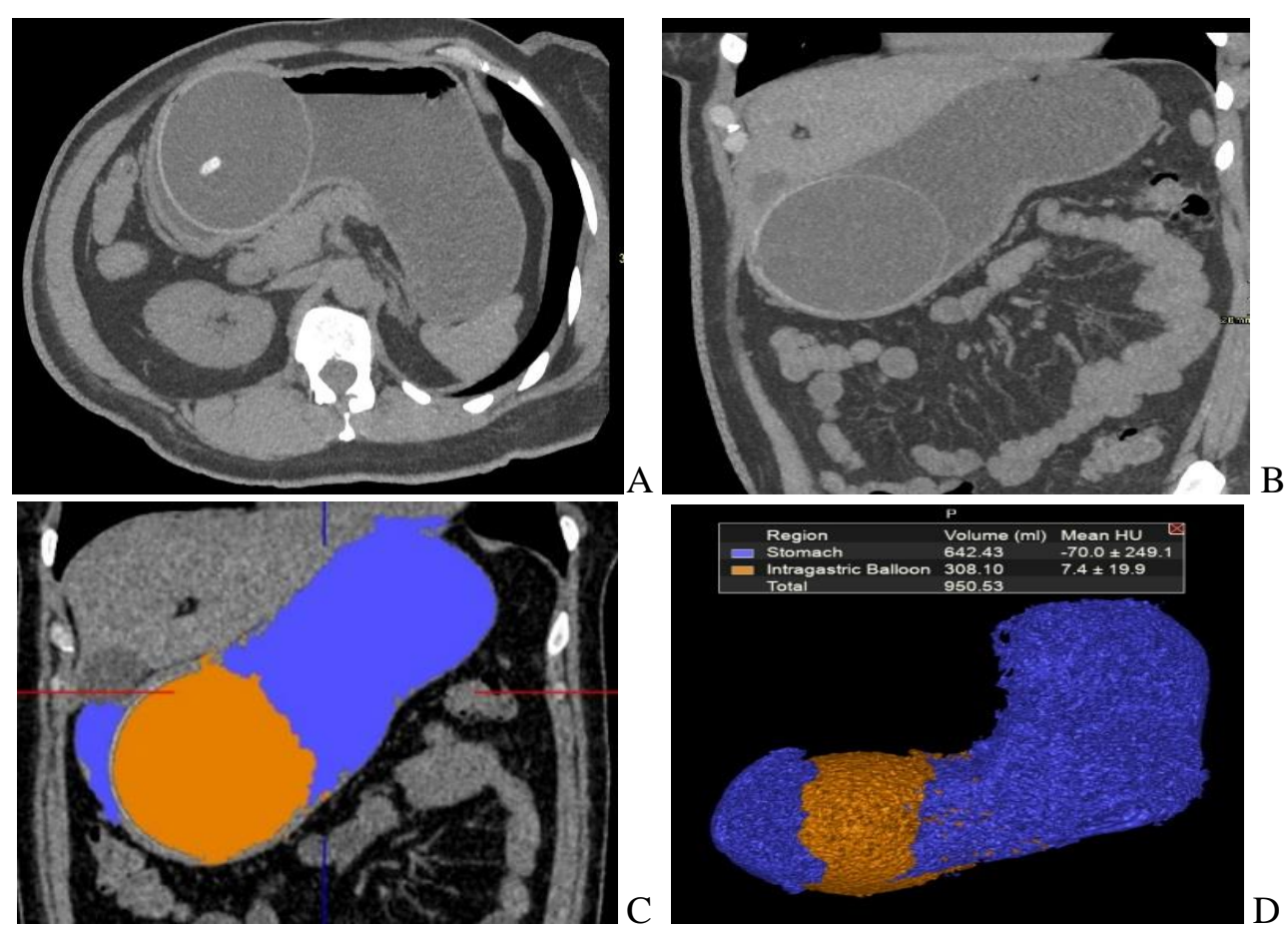

Figure (11): A 46 year old female patient with history of placed intragastric balloon 9 months earlier who experienced central and right upper abdominal pain, distension and discomfort with food intolerance, nausea and recent non bilious vomiting. (A and B) Reformatted axial and coronal oblique images and (C) Colored overlaid coronal image of nonenhanced CT showing the fluid filled intragastric balloon within the antrum that measured $9 \mathrm{~cm}$ in diameter and the 
proximal stomach is moderately distended by large amount of the ingested water and showing air fluid level with no food particles seen. There was a little amount of fluid seen distal to the balloon that passed around it. (D) 3D volumetric image showing the intragastric balloon in orange color with calculated volume of $\pm 308 \mathrm{cc}$ and the rest of the stomach in blue color with calculated volume of $\pm 950 \mathrm{cc}$. Picture of gastric outlet obstruction secondary to intragastric balloon.

\section{DISCUSSION}

Bariatric operations are generally safe and effective, but they result in permanent alteration of gastric anatomy, which may lead to complications at any time during the course of a patient's life (16). Physical examination of these patients is impaired by obesity itself and so, comes the value of imaging studies and the importance of the radiologist to be familiar with any expected postoperative complications (17). According to Lim et al ${ }^{(\mathbf{1 6})}$, these problems may present either early or late in the postoperative course and they depend on the type of operation.

CT scan is the best investigation modality for any patient with any postoperative abdominal symptoms as mentioned by Srikanth $\boldsymbol{e t} \boldsymbol{a l} .^{(18)}$ as it can identify collections, bowel obstruction, internal and port site hernias and intussusception beside its ability in the assessment of bowel wall thickening and fistulas.

In our study, 275 patients who were suspected to have post non sleeve bariatric procedures complication underwent radiological examination either with UGI fluoroscopy or CT or both. Out of these patients, 195 (70.9\%) had done RYGB, 76 (27.6\%) had done LAGB and the remaining four had placed intra-gastric balloon.

Out of the 195 patients who had done RYGB, we observed various complications in 21 patients $(10.8 \%)$, most of them (19 patients; 90.5\%) developed late complications. In those underwent LAGB, 8 out of 76 patients $(10.5 \%)$ developed complications, which were all late ones. Lastly, Out of the 4 patients who had intragastric balloon, radiological findings of complication were found in 2 patients and we suggested a spontaneously relieved complication in a third one.

\section{Roux-en-Y gastric bypass (RYGB) complications}

Ribas et al. ${ }^{\left({ }^{17}\right)}$, found abnormal CT findings in 10 out of 40 (25\%) patients undergoing laparoscopic RYGB operation, one $(2.5 \%)$ with gastric stenosis, 5 (12.5\%) with internal hernia, one (2.5\%) with anastomotic leak and $3(7.5 \%)$ with abscess. While, Blachar et al. ${ }^{(19)}$ found complications in $9.5 \%$ of patients undergoing RYGB, which is comparable to our results.

Of these complications, extraluminal leak is the most serious early one occurring in $2-5 \%$ of patients within 10 days of surgery. Leaks may be small and selfcontained or large and potentially life threating ${ }^{(\mathbf{2 0}, 21)}$. In our patients following RYGB, 2 (1\%) showed extraluminal leakage from the gastrojejunal anastomosis. This is going with the report of Ribas $\boldsymbol{e t}$ al. ${ }^{(17)}$, who found leakage in one of their 40 patients (2.5\%) underwent RYGB. Our 2 patients with leakage were managed by nasojejunal tube inserted under fluoroscopy for feeding to bypass the gastric pouch and external percutaneous drainage either by US-guided pigtail insertion into left subphrenic space or by surgical drains. Gastric stent was placed in 1 of them to help seal the dehiscence.

Unfortunately, those 2 patients developed abscess (1\%). In fact, Shah et al. ${ }^{(5)}$ stated that subphrenic abscess occur in fewer than $2 \%$ of all bariatric surgery patients, and this is extremely serious complications with a high morbidity and mortality. However, others as Ribas et al. ${ }^{(17)}$ found higher incidence of abscess (7.5\%), which may be due to technical sepsis in the operation.

A fistula between the gastric pouch and the excluded stomach had developed in one of our patients with abscess $(0.5 \%)$ as previously mentioned by Riaz et al. ${ }^{(20)}$ who mentioned that fistula is seen in less than 1 $\%$ of patients. In fact, CT signs of leakage include direct signs with extraluminal oral contrast visualization and indirect ones as visualization of perigastric pouch collections and abdominal cavity free fluid ${ }^{(\mathbf{1 7})}$. In our study, leakage was near the gastrojejunal anastomosis and accumulated on its left side and extended to the left subphrenic area, which is in agreement with Levine and Carucci ${ }^{(21)}$, who stated that leakage is usually located at the gastrojejunostomy but can also be present at the gastric pouch, jejunal stump, and jejunojejunal anastomosis.

Intestinal obstruction was observed in 4 of our patients $(2 \%)$ that caused by internal hernia in one patient, early postoperative port site incarcerated hernia in another case and intussusception in the other two cases. It was mentioned that small bowel obstruction (SBO) occurs in up to $5 \%$ of patient, usually due to different factors as adhesions, internal hernia, anterior abdominal wall hernias, jejunojejunal anastomotic strictures, and intussusception ${ }^{(22)}$.

Regarding internal hernia, despite being considered as a late complication, it can occur at any time after the surgery. It usually results from small bowel herniation into a defect either in the transverse mesocolon (for a retrocolic alimentary limb) or in the small bowel mesentery at the jejunojejunal anastomosis or posterior to the alimentary Roux limb (Petersen defect). SBO can occur from small bowel incarceration in the internal hernia with potential infarction and perforation that could be fatal if not managed promptly ${ }^{(22)}$. Actually, the most frequent complication we observed in our study was internal hernia that occurred in 14 patients $(7.2 \%)$, one of them $(7.1 \%)$ developed intestinal obstruction and it was associated with intussusception in another one (7.1\%). Ribas et al. ${ }^{\left({ }^{17}\right)}$ also found internal hernia as the most frequently encountered complication in their study, 5 patients out of 40 (12.5\%) with different CT signs including rotation of the mesenteric vessels (whirl sign), presence of a herniated jejunal segment located 
above the gastric level, mesenteric fat planes densification, and/or mushroom appearance of the distended loops in the left hypochondrium.

In addition, we encountered intussusception in 3 of our cases $(1.5 \%)$ that led to intestinal obstruction in two of them $(66.7 \%)$ and was associated with internal hernia in 1 case $(33.3 \%)$. In fact, intussusceptions in adults are often transient, non-obstructing, and rarely cause SBO. However, after RYGB procedure, intussusceptions, either transient or fixed, can occur at both the gastrojejunal and jejunojejunal anastomosis with incidence of $0.4 \%$, due to ectopic peristaltic pacing tissue in the divided jejunum triggering dysfunctional retrograde contractions or due to the anastomotic staple line acting as a trigger ${ }^{(\mathbf{2 0}, \mathbf{2 1})}$.

Gastrojejunal anastomotic inflammatory changes were noticed in 1 of our patients $(0.5 \%)$ with jejunal marginal mucosal ulceration. Gastrojejunostomy marginal ulcers were reported in 3\%-13\% of patients by Levine and Carucci (21). Rasmussen et al. (23) suggested that prolonged exposure of alimentary jejunal limb to the gastric acid juice is the cause of its occurrence.

Regarding fistula between the gastric pouch and the excluded stomach (gastrogastric), it was observed in 3 of our patients $(1.5 \%)$ and in one case it occurred after leakage. Actually, gastrogastric fistula is uncommon complication and seen in $<1-3.7 \%$ of patients after RYGB, occurring at early and late periods ${ }^{(20)}$ that is mainly associated with staple line dehiscence in early phases and extraluminal leaks, and it may lead to recurrent weight gain in its late incidence ${ }^{\text {(24). }}$.

Lastly, we also observed hiatus hernia in 3 cases $(1.5 \%)$ which is going with previous authors who mentioned that bariatric surgery (RYGB) dramatically alters normal stomach anatomy resulting in a significant incidence of hiatal hernia and gastroesophageal reflux disease ${ }^{(25)}$.

In our study, the two cases of postoperative leak were diagnosed by both upper GI series and CT examination, which is similar to Al Hajj and Chemaly (26) statement that $100 \%$ of cases of leak/fistula were discovered by upper GI series and CT study. However, Latif et al. ${ }^{(27)}$ reported in their study $70 \%$ sensitivity of upper GI series in diagnosis of post-bariatric surgery complication concluding higher sensitivity of the CT in leak detection over upper GI series.

Lastly, we found that upper GI series has no role in diagnosing complications such as abscess, internal hernia, port site hernia and intussusception or revealing the cause of intestinal obstruction, where CT scan was able to diagnose. Thus, as compared to UGI series, CT showed superior role in post RYGB complication detection, which is consistent with Bassiouny and Chalabi ${ }^{(28)}$ result who reported higher CT sensitivity series in in the detection of all post RYGB complications.
Laparoscopic Adjustable Gastric Banding (LAGB):

Despite being minimally invasive procedure, early and delayed complications can occur after LAGB that may lead to its dysfunction. Early complications are rare including gastric perforation, infection and malposition of band, while most complications are delayed and comprise gastroesophageal reflux, pouch dilatation, band slippage and intragastric band erosion. Other possible delayed complications are esophageal dysmotility and dilatation, small bowel obstruction, port-site infection and disconnection of its components (29).

In our study, we encountered post LAGB complications in 8 of 76 patients $(10.5 \%)$. According to Carucci et al. ${ }^{(30)}$ there is a wide frequency range from 1.4 up to $26 \%$ of the reported complications including its components (the band, connector tube and reservoir port).

We observed band slippage in 5 patients $(6.6 \%)$, which was associated with gastric wall band erosion in 1 case and in another case retro-peritoneal hematoma occurred after its removal. Distal band slippage is a relatively common complication occurring in 3\%-13\% of patients leading to eccentric pouch dilatation and acute symptoms due to stomal obstruction ${ }^{(\mathbf{5}, \mathbf{2 0})}$.

Intragastric erosion and band migration are late rare complications $(<2 \%)$ and could be attributed to gastric wall pressure necrosis by the high pressures of the inflated band according to Nocca et al. ${ }^{(31)}$. We observed band erosion in 1 case of our study (1.3\%) and it was associated with slippage.

Pouch dilatation was found in 6 of our cases (7.9\%) and band slippage was the cause in 5 of them (83.3\%). In the remaining case, the cause was dietary noncompliance with overfilling as the band was in normal position and the dilated pouch formed paraesophageal hiatus hernia was associated with megaesophagus and pseudo-achalasia. This is going with previous result of 3\%-8\% found by Levine et al. ${ }^{(21)}$, who mentioned that pouch dilatation may be chronic with normal or even widened stoma due to failure to modify eating habits after band placement or acute due to marked stomal narrowing mandating immediate band deflation.

Regarding mechanical port/tubing complications, infection is considered the commonest device-related complication affecting up to $6 \%$ of patients ${ }^{(30)}$. CT examination is valuable in defining the superficial inflammatory changes with any possible abscess formation and intra-abdominal spread of infection into the peritoneum or stomach ${ }^{(29)}$. In addition, Riaz et al. (20) stressed the importance of observing disconnection which could happens anywhere through the port, tubing, or the band and lead to system failure, as was observed in 2 of our cases $(2.6 \%)$.

\section{Gastric Balloon:}


While minimally invasive procedure, patients with gastric balloon may complain from intolerance in about 7 to $9 \%$ necessitating early removal ${ }^{(\mathbf{1 3})}$ and up to $5.5 \%$ of them develop complications that may be mild as abdominal discomfort, gastroesophageal reflux, nausea and vomiting or significant including, rupture and migration of the balloon $(0.36 \%)$, bleeding ulcers $(0.2 \%)$, gastric outlet obstruction $(0.76 \%)$ and gastric perforation ${ }^{(13,14)}$.

The newer generations of balloons are filled with saline to induce more effective weight loss and methylene blue to stain the urine in case of balloon rupture or deflation, facilitating early detection by the patient. With newer generations of intragastric balloons, the majority of the encountered significant complications occur after 6 months of its placement ${ }^{(15)}$.

The reported important complications include balloon migration to the bowel after spontaneous deflation that may need surgical removal, gastric ulcers and gastric outlet obstruction, which may lead to necrosis and perforation ${ }^{(13)}$. The deflated balloon, sometimes pass the bowels without any complication (32).

We observed in our study one case of spontaneously deflated neglected intragastric balloon that distally migrated till proximal ilium causing small intestinal obstruction. In another patient, despite the one day severe symptoms of colicky pain, vomiting and diarrhea with urine bluish discoloration, the balloon wasn't seen at all within the stomach or the bowel loops and a suggestion of balloon spontaneous deflation and distal migration passing out of the alimentary canal was made with no residual complication based on the negative radiological finding.

Regular follow up of the intragastric balloon size by ultrasound for early detection of possible spontaneous deflation and distal migration could obviate the need for the surgery ${ }^{(33)}$.

Also, we observed one case with gastric outlet obstruction secondary to the intragastric balloon seen distending gastric antrum that was endoscopically deflated and removed in the next day. This is in agreement with Koek and Hammond ${ }^{(14)}$ who stated that gastric outlet obstruction induced by intragastric balloon requires endoscopic balloon deflation and removal after initial resuscitation. Genco et al. (13) reported gastric outlet obstruction secondary to intragastric balloons within the first two weeks in $0.76 \%$ of their cases. This situation may lead to more serious complications with progression to gastric wall necrosis and perforation that necessitates early diagnosis and urgent intervention ${ }^{(34)}$. Abdominal CT helps in diagnosis of balloon induced gastric outlet obstruction and any further progression to necrosis ${ }^{(\mathbf{1 4})}$.

\section{CONCLUSION}

It could be concluded that bariatric procedures may be followed by many complications and accurate diagnosis of these problems by proper radiological procedures as MSCT beside fluoroscopy is imperative. In addition, radiologists must be aware and well trained for detection of new postoperative anatomy and radiological features of all these possible complications.

Financial support and sponsorship: Nil.

Conflict of interest: Nil.

\section{REFERENCES}

1. Alebshehy R, Shuaib N, Mbako J et al. (2016): Determinant analysis of obesity among adult females in Egypt. Egyptian Journal of Hospital Medicine, 65(1): 662-669.

2. Clayton R, Carucci L (2018): Imaging following bariatric surgery: roux-en-Y gastric bypass, laparoscopic adjustable gastric banding and sleeve gastrectomy. Br J Radiol., 91(1089): 20180031. doi: 10.1259/bjr.20180031

3. Afshin A, Forouzanfar M, Reitsma M et al. (2017): Health effects of overweight and obesity in 195 countries over 25 years. N Engl J Med., 377(1): 13-27.

4. Abdelkarim O, Ammar A, Trabelsi K et al. (2020): Prevalence of underweight and overweight and its association with physical fitness in Egyptian schoolchildren. Int J Environ Res Public Health, 17(1): 75-85.

5. Shah S, Shah V, Ahmed A et al. (2011): Imaging in bariatric surgery: Service set-up, post-operative anatomy and complications. Br J Radiol., 84: 101-111.

6. Azab E (2018): The role of MDCT study to assess postoperative acute complications of laparoscopic sleeve gastrectomy (LSG): Egypt J Radiol Nucl Med., 49: 948952.

7. Sullivan S, Kumar N, Edmundowicz S et al. (2015): ASGE position statement on endoscopic bariatric therapies in clinical practice. Gastrointestinal Endoscopy, 82(5): 767-772.

8. Cummings D, Overduin J, Foster-Schubert K (2004): Gastric bypass for obesity: mechanisms of weight loss and diabetes resolution. J Clin Endocrinol Metab., 89(6): 2608-2615.

9. Korenkov M, Sauerland S (2007): Clinical update: Bariatric surgery. Lancet, 370: 1988-1990.

10. Herron D, Roohipour R (2012): Complications of Roux-en-Y gastric bypass and sleeve gastrectomy. Abdom Imaging, 37: 712-718. doi:10.1007/s00261-0129866-6

11. Gaetke-Udager $K$, Wasnik A, Kaza $R$ et al. (2014): A guide to imaging in bariatric surgery. Emerg Radiol., 21(3): 309-319.

12. Mehanna M, Birjawi G, Moukaddam H et al. (2006): Complications of adjustable gastric banding, a radiological pictorial review. Am J Roentgenol., 186(2): 522-534.

13. Genco A, Bruni T, Doldi S et al. (2005): BioEnterics intragastric balloon: The Italian experience with 2,515 patients. Obes Surg., 15: 1161-1164.

14. Koek S, Hammond J (2018): Gastric outlet obstruction secondary to orbera intragastric balloon. J of Surgical Case Reports, 10: 1-3.

15. Stavrou G, Tsaousi G, Kotzampassi K (2019): Lifethreatening visceral complications after intragastric 
balloon insertion: Is the device, the patient or the doctor to blame? Endosc Int Open, 7(2): 122-129.

16. Lim R, Beekley A, Johnson D et al. (2018): Early and late complications of bariatric operation. Trauma Surg Acute Care Open, 3: 219-224.

17. Ribas F, Nassif P, Ribas C et al. (2012): Postoperative abdominal CT findings in patients submitted to Roux-eny gastric bypass without ring. Rev Col Bras Cir., 39(3): 189-194.

18. Srikanth M, Keskey T, Fox S et al. (2004): Computed tomography patterns in small bowel obstruction after open distal gastric bypass. Obes Surg., 14(6): 811-822.

19. Blachar A, Federle $M$, Pealer $K$ et al. (2002): Gastrointestinal complications of laparoscopic Roux-en$\mathrm{Y}$ gastric bypass surgery: clinical and imaging findings. Radiology, 223(3): 625-632.

20. Riaz R, Myers D, Williams T (2016): Multidetector CT imaging of bariatric surgical complications: a pictorial review. Abdom Radiol (NY), 41(1): 174-188.

21. Levine $M$, Carucci $L$ (2014): Imaging of bariatric surgery: Normal anatomy and postoperative complications. Radiology, 270 (2): 327-341.

22. Carucci L, Turner M, Shaylor S (2009): Internal hernia following Roux-en-Y gastric bypass surgery for morbid obesity: Evaluation of radiographic findings at small-bowel examination. Radiology, 251 (3): 762-770.

23. Rasmussen J, Fuller W, Ali M (2007): Marginal ulceration after laparoscopic gastric bypass: an analysis of predisposing factors in 260 patients. Surg Endosc., 21(7): 1090-1094.

24. Carucci L, Conklin R, Turner M (2008): Roux-en-Y gastric bypass surgery for morbid obesity: evaluation of leak into excluded stomach with upper gastrointestinal examination. Radiology, 248 (2): 504-510.

25. Flanagin B, Mitchell $M$, Thistlethwaite $\mathrm{W}$ et al. (2010): Diagnosis and treatment of atypical presentations of hiatal hernia following bariatric surgery. Obes Surg., 20(3): 386-392.
26. Al Hajj G, Chemaly $R$ (2018): Fistula following laparoscopic sleeve gastrectomy: a proposed classification and algorithm for optimal management. Obesity Surg., 28:656-664.

27. Latif M, Fouda N, Omran E et al. (2020): Role of imaging in assessment and detection of complications after bariatric surgery. Egyptian Journal of Radiology and Nuclear Medicine, 51: 41-46.

28. Bassiouny R, Chalabi N (2020): Value of contrastenhanced multidetector computed tomography in imaging of symptomatic patients after laparoscopic Roux-en-Y gastric bypass and laparoscopic sleeve gastrectomy. Egyptian Journal of Radiology and Nuclear Medicine, 51: 25-32.

29. Sonavane S, Menias C, Kantawala $K$ et al. (2012): Laparoscopic adjustable gastric banding: What radiologists need to know? Radio Graphics, 32: 11611178.

30. Carucci L, Turner M, Szucs R (2007): Adjustable laparoscopic gastric banding for morbid obesity: imaging assessment and complications. Radiol Clin N Am., 45 (2): 261-274.

31. Nocca D, Frering V, Gallix B et al. (2005): Migration of adjustable gastric banding from a cohort study of 4236 patients. Surg Endosc., 19(7): 947-950.

32. Doldi S, Micheletto G, Perrini M et al. (2002): Treatment of morbid obesity with intragastric balloon in association with diet. Obesity Surgery, 12 (4): 583-587.

33. Francica G, Giardiello C, Iodice G et al. (2004): Ultrasound as the imaging method of choice for monitoring the intragastric balloon in obese patients: normal findings, pitfalls and diagnosis of complications. Obesity Surgery, 14(6): 833-837.

34. Tate C, Geliebter A (2017): Intragastric balloon treatment for obesity: Review of recent studies. Adv Ther., 34:1859-75. 